\title{
Intermittent boundary layers and torque maxima in Taylor-Couette flow
}

\author{
Hannes J. Brauckmann ${ }^{1}$ and Bruno Eckhardt ${ }^{1,2}$ \\ ${ }^{1}$ Fachbereich Physik, Philipps-Universität Marburg, D-35032 Marburg, Germany \\ ${ }^{2}$ J.M. Burgerscentrum, Delft University of Technology, 2628 CD Delft, The Netherlands
}

(Received 22 October 2012; published 8 March 2013)

\begin{abstract}
Turbulent Taylor-Couette flow between counter-rotating cylinders develops intermittently fluctuating boundary layers for sufficient counter-rotation. We demonstrate the phenomenon in direct numerical simulations for radius ratios $\eta=0.5$ and 0.71 and propose a theoretical model for the critical value in the rotation ratio. Numerical results as well as experiments show that the onset of this intermittency coincides with the maximum in torque. The variations in torque correlate with the variations in mean Taylor vortex flow, which is first enhanced for weak counter-rotation and then is reduced as intermittency sets in. To support the model, we compare it to numerical results, to experiments at higher Reynolds numbers, and to Wendt's data.
\end{abstract}

DOI: 10.1103/PhysRevE.87.033004

PACS number(s): 47.20.Qr, 47.27.N-

\section{INTRODUCTION}

The flow between concentric cylinders has served as a paradigm for the transition to turbulence since Taylor's 1923 characterization of the bifurcation from laminar to vortical flows [1]. Many transitions between spatially and temporally simple flow states, such as vortices, modulated vortices, and traveling waves, are accessible by standard bifurcation theory, and have been described and studied in considerable detail $[2,3]$. The turbulent states that are reached after several of these bifurcations are not always homogeneous but can show patchiness in the form of turbulent spots or turbulent spirals [4-7]. In addition to such azimuthal and axial modulations, Coughlin and Marcus [8] have described a radial inhomogeneity for counter-rotating cylinders, which consists of turbulent bursts that have been seen in experiments by Colovas and Andereck [9]. Measurements in the Twente Taylor-Couette facility have confirmed the presence of this inhomogeneity up to Reynolds numbers of $10^{6}$ [10]. Moreover, in Ref. [10], the onset of this instability has been linked to the maximum in torque that appears for moderate counter-rotation [11-14].

Coughlin and Marcus [8] already suggested that this radial inhomogeneity should be linked to the presence of a neutral surface of vanishing angular velocity in the laminar Couette profile for counter-rotating cylinders. The region between the inner cylinder and the neutral surface is inviscidly unstable by the Rayleigh criterion, whereas, the region between the neutral surface and the outer cylinder is Rayleigh stable. These considerations do not provide an immediate prediction for the onset of inhomogeneity since the neutral surface already appears with infinitesimal amounts of counter-rotation. Here, we discuss the extensions needed in order to derive a predictive theory for the onset of this intermittency, compare it with observations for different radius ratios, and describe the link to the torque maxima.

The outline of the paper is as follows. In Sec. II, we introduce the numerical simulations and describe the phenomenon. We also identify the onset of intermittency and summarize numerical and experimental results for torque maxima, including a reanalysis of the data of Wendt [15], which is documented in the Appendix. In Sec. III, we present the argument for the boundary layer intermittency, and in
Sec. IV, we describe the link to the torque maximum. We conclude with a few remarks in Sec. V.

\section{BOUNDARY LAYER INTERMITTENCY}

In order to introduce the phenomenon, we present results from direct numerical simulations for different radius ratios. We solve the incompressible Navier-Stokes equation with the spectral scheme explained in Ref. [16]. For the dimensionless units, we measure all lengths and times in units of the gap width $d=r_{o}-r_{i}$, where $r_{o}$ and $r_{i}$ are the radii of the outer and inner cylinders, respectively, and the viscous time $d^{2} / v$ with the kinematic viscosity $v$. To avoid the end effects caused by top and bottom lids in experiments, an additional periodicity in the axial direction of length $L_{z}$ is introduced, resulting in an aspect ratio $\Gamma=L_{z} / d$. Here, we select $\Gamma=2$, which allows for one Taylor vortex pair when the outer cylinder is at rest. Fourier modes and Chebyshev polynomials are employed for expansions in the two periodic and the wall-normal directions, respectively. We simulate a domain of reduced azimuthal length, i.e., one third for $\eta=r_{i} / r_{o}=0.5$ and one ninth for $\eta=0.71$ of the full azimuthal length. Consequently, the flow field repeats three (nine) times to fill the entire circumference. We tested that the shorter azimuthal period does not influence the computed torques for a stationary outer cylinder. The criteria used to test and to verify the code are detailed in Ref. [13]. Specifically, the spatial resolution, characterized by the number of modes $\left(N_{z}, N_{\varphi}, N_{r}\right)$ in each direction, is chosen so that three convergence criteria are satisfied: Torque computed at the inner and outer cylinders has to agree within a relative deviation of $5 \times 10^{-3}$, the expansion coefficients in each direction have to cover a range of, at least, $10^{4}$, and the energy dissipation estimated from the torque has to agree with the volume energy dissipation rate to within $10^{-2}$. All these requirements are met in all simulations shown here.

We consider radius ratios $\eta=0.5$ and $\eta=0.71$. In order to assess the influence of the mean system rotation on turbulent characteristics, the simulations are performed at a fixed shear between the cylinder walls, defined by Dubrulle et al. [17] as

$$
\operatorname{Re}_{S}=\frac{2}{1+\eta}\left|\eta \operatorname{Re}_{o}-\operatorname{Re}_{i}\right|,
$$


with $\operatorname{Re}_{i}=\left(r_{o}-r_{i}\right) r_{i} \omega_{i} / v$ and $\operatorname{Re}_{o}=\left(r_{o}-r_{i}\right) r_{o} \omega_{o} / \nu$. Here, $\omega_{i}$ and $\omega_{o}$ denote the angular velocities of the inner and outer cylinders, and $v$ is the kinematic viscosity of the fluid. For both radius ratios, we realize various mean rotations characterized by the rotation ratio $\mu=\omega_{o} / \omega_{i}$ for the same shear $\operatorname{Re}_{S}=$ $2.0 \times 10^{4}$. According to Lathrop et al. $[18,19]$, this value is high enough for the flow to become fully turbulent for $\mu=0$ so that the torque increases with $\operatorname{Re}_{i}$ as in other turbulent wall-bounded shear flows. The dimensionless torque $G$ exerted on the inner and outer cylinders is obtained from

$$
G(t)=v^{-2} J^{\omega}=v^{-2} r^{3}\left(\left\langle u_{r} \omega\right\rangle_{A(r)}-v \partial_{r}\langle\omega\rangle_{A(r)}\right),
$$

where $u_{r}$ and $\omega=u_{\varphi} / r$ denote the radial and angular velocities, respectively, and $\langle\cdots\rangle_{A(r)}$ stands for an area average over the surface of a concentric cylinder [20]. The long-time mean value is obtained from an additional average over time.

For corotation, i.e., $\mu \geqslant 0$, torque values at the inner and outer cylinders agree on average and in their fluctuations [13]. However, the situation drastically changes for strong counter-rotation as illustrated by the torque time series for $\mu=-0.5$ in Fig. 1(a). Near the inner cylinder, fluctuations are small and without long-time variation. At the outer cylinder, the torque exhibits fluctuations of relatively strong amplitude and slow dynamics that qualitatively differ from the ones at the
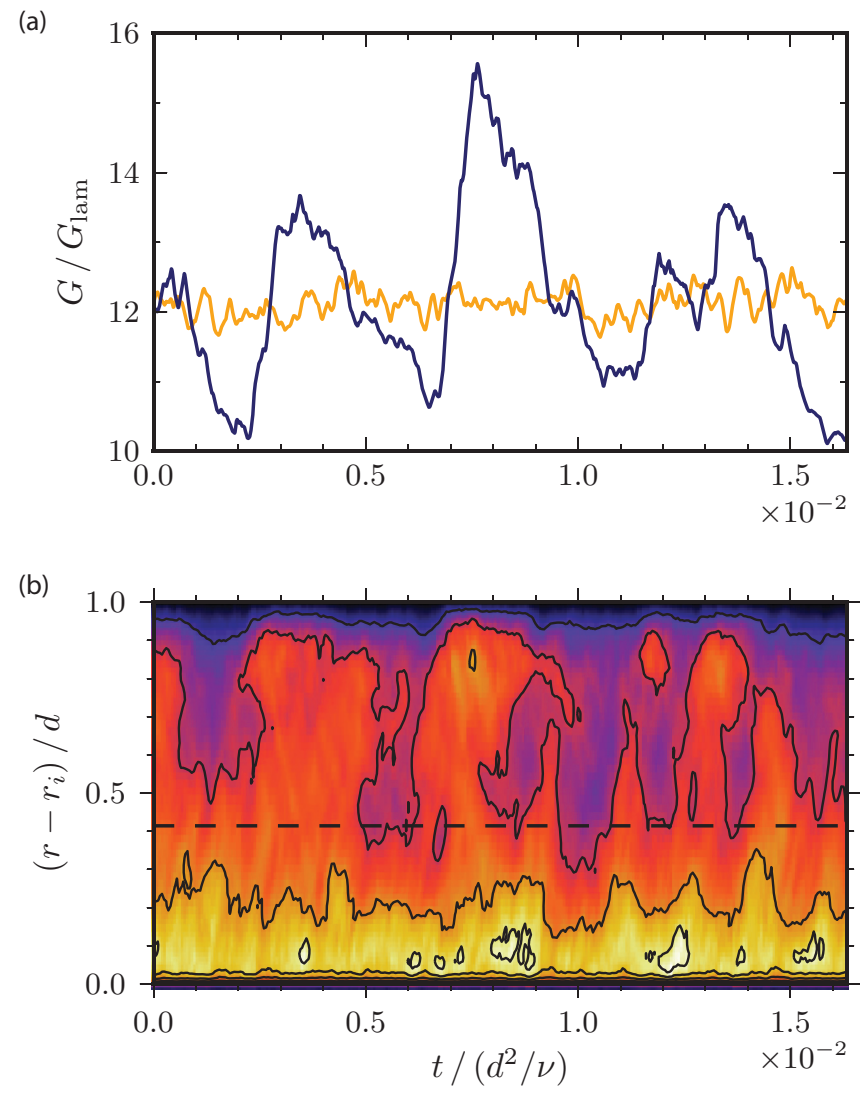

FIG. 1. (Color online) Turbulent bursts for $\eta=0.5, \operatorname{Re}_{S}=$ 20000 , and $\mu=-0.5$. (a) Time series of the torque divided by its laminar value $G_{\text {lam }}$. Dark line (blue): outer cylinder. Gray line (orange): inner cylinder. (b) Spatiotemporal plot of cross-flow energy $E_{c f}(r, t)$ averaged along the periodic coordinates with white (black) corresponding to maximum (minimum) energy. The horizontal dashed line indicates the laminar neutral surface. inner cylinder. These slow fluctuations reflect an intermittent turbulent activity in the vicinity of the outer cylinder as demonstrated by the cross-flow energy in Fig. 1(b). The cross-flow energy,

$$
E_{c f}(r, t)=\left\langle u_{r}^{2}+u_{z}^{2}\right\rangle_{A(r)}
$$

measures the energy content in the transverse velocity components at a radial distance $r$ and at an instant of time. Small values indicate a flow close to laminar; large values indicate a turbulent state. One notes that, near the inner cylinder, the flow is more or less homogeneously turbulent, whereas, towards the outer cylinder, one observes an increased activity synchronized with the increase in mean torque.

For this geometry, the Rayleigh-stable region of the laminar profile extends over the interval $\left(r-r_{i}\right) / d \in[0.414,1]$ with the lower bound marked by a dashed line in Fig. 1(b). The presence of the bursts shows that this stability is not maintained, but the position of the laminar line still marks the transition between small radial variations near the inner cylinder and larger ones towards the outer cylinder.

For $\operatorname{Re}_{S} \sim 2.3 \times 10^{3}$, Coughlin and Marcus [8] described the bursts as instability of spatially ordered "interpenetrating spiral" flow. Here, we observe turbulent flow in the Rayleighunstable inner region at $\operatorname{Re}_{S}=2.0 \times 10^{4}$, that is accompanied by intermittent turbulence in the outer one. The connection to spirals cannot be followed up because of the limited axial and azimuthal domain sizes that can be computed. The experiments of Ref. [10] extend this observation to even larger Reynolds numbers $\operatorname{Re}_{S} \sim 10^{6}$ where the presence of turbulent bursts in the outer region is deduced from a bimodal distribution of the angular velocity.

The onset of the intermittent behavior is accompanied by an increase in the torque fluctuations. Therefore, we study the standard deviation $\sigma_{G}$ of the torque relative to the mean for the outer cylinder, i.e., the ratio $\sigma_{G} / G$ as an indicator and deduce a critical value $\mu_{c}(\eta)$ for the onset from the requirement that $\sigma_{G} / G$ exceeds the base level for $\mu=0$, cf. Fig. 2. The choice of $\sigma_{G} / G$ as a measure for the transition is supported by the observation that it remains relatively unaffected by variations in $\mu$ at the inner cylinder. For the numerical simulations, we find the critical values,

$$
\begin{aligned}
\mu_{c}(0.5) & =-0.208 \pm 0.014, \\
\mu_{c}(0.71) & =-0.325 \pm 0.050
\end{aligned}
$$

marked by dashed lines in Fig. 2. The uncertainties are estimated as half the gap in $\mu$ between the two data points next to the critical value. The critical point varies with $\eta$, and we return to this feature in Sec. III. The onset of intermittent bursts was determined experimentally at $\mu_{c}(0.716) \approx-0.368$ for $\operatorname{Re}_{S} \sim 10^{6}[10]$. The difference between this value and our observation (4) may be due to our uncertainty in $\mu_{c}$ or the difference in Reynolds number, but it is not significant.

The rotation ratio $\mu_{\max }(\eta)$ of maximal torque was first identified independently in two experiments [10-12]. For a constant shear, they report a torque maximization for $\mu_{\max }(0.716)=-0.33 \pm 0.04$ [10] and for $\mu_{\max }(0.7245)=$ -0.333 [12]. Numerical simulations reveal that the torque maximum for counter-rotation only occurs after a shift in the maximizing $\mu$ value with increasing $\operatorname{Re}_{S}$ [13]. Figure 3 

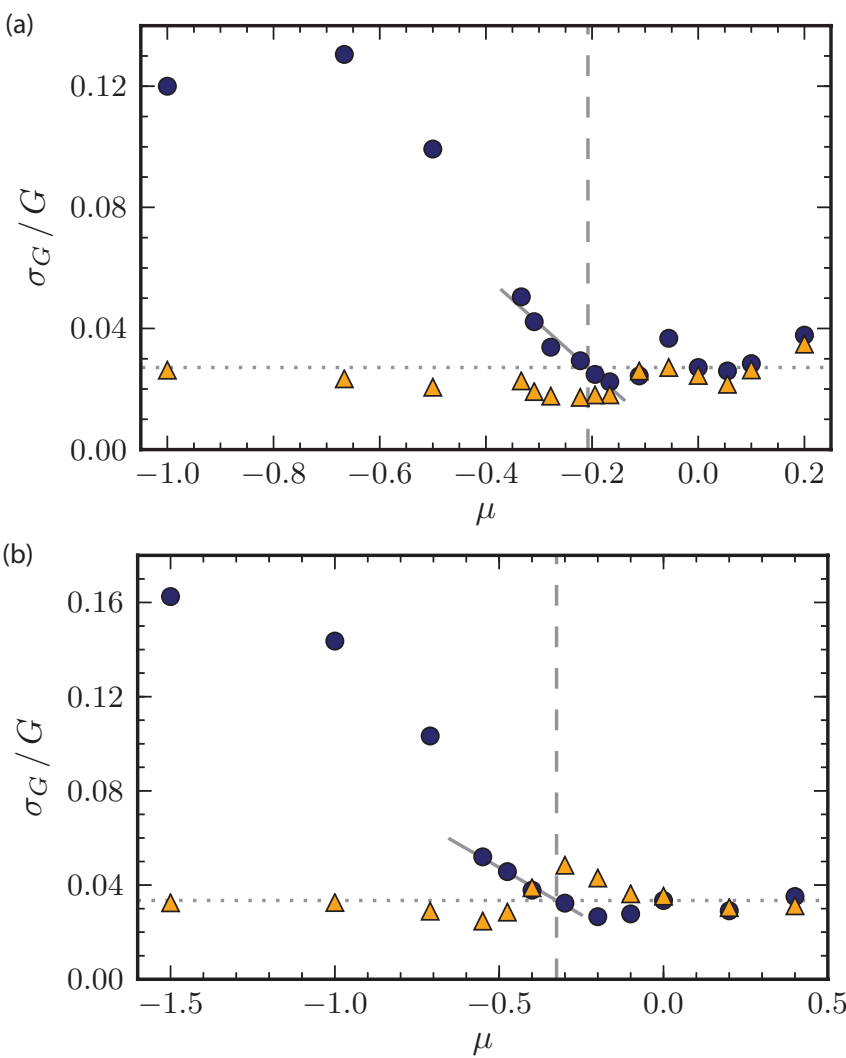

FIG. 2. (Color online) Standard deviation $\sigma_{G}$ (divided by the mean $G$ ) of temporal torque fluctuations for a constant shear and various global rotations. Dark circles (blue): calculated at the outer cylinder. Gray triangles (orange): calculated at the inner cylinder. The dotted line marks the fluctuation base level for $\mu=0$, and the solid line is a linear fit to six points in (a) and to four points in (b) through which the line runs. The dashed line indicates the intersection point $\mu_{c}$ given in Eq. (4). (a) $\eta=0.5$ and $\operatorname{Re}_{S}=20000$. (b) $\eta=0.71$ and $\operatorname{Re}_{S}=19737$.

shows the computed torques for $\operatorname{Re}_{S}=2.0 \times 10^{4}$ just at the beginning of the asymptotic regime. We determine the rotation ratio of optimal transport as the maximum of a quadratic fit $G / G_{\text {lam }}=c_{2} \mu^{2}+c_{1} \mu+c_{0}$ to five data points and find

$$
\begin{gathered}
\mu_{\max }(0.5)=-0.195 \pm 0.019, \\
\mu_{\max }(0.71)=-0.357 \pm 0.060 .
\end{gathered}
$$

The uncertainties are deduced from the relative confidence interval $\Delta G / G$, which results from temporal torque fluctuations. This uncertainty in the torque values transforms into an uncertainty in the maximum location of the quadratic fit, i.e.,

$$
\Delta \mu_{\max }=\sqrt{-\frac{\Delta G}{c_{2} G}\left(G / G_{\mathrm{lam}}\right)_{\max }},
$$

with the fit coefficient $c_{2}$ and the maximal rescaled torque $\left(G / G_{\text {lam }}\right)_{\max }$.

For both radius ratios, the maximizing global rotation $\mu_{\max }$ compares well with the transition to the radial inhomogeneity at $\mu_{c}$ as previously observed experimentally by van Gils et al. [10] for $\eta=0.716$. On the other hand, the critical values for $\eta=0.71$ are more uncertain, which complicates the identification of a correspondence between them. We note

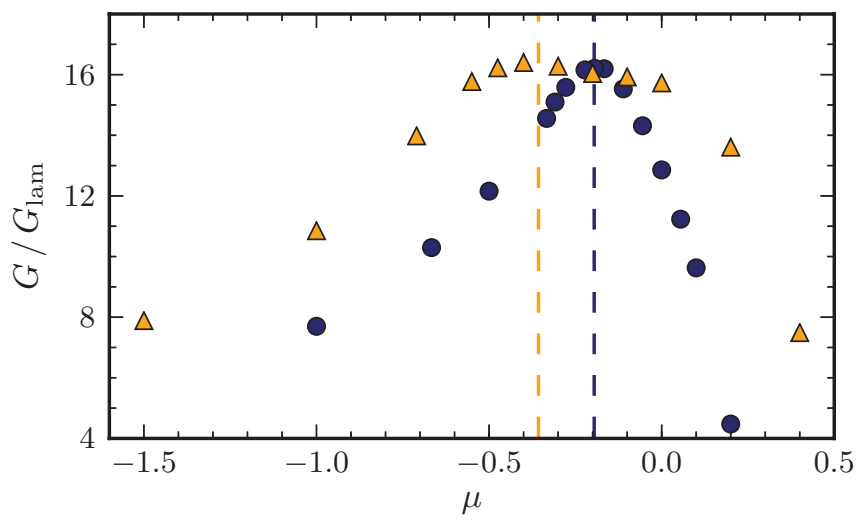

FIG. 3. (Color online) Dependence of the total torque on the global rotation. Dark circles (blue): $\eta=0.5$ and $\operatorname{Re}_{S}=20000$. Gray triangles (orange): $\eta=0.71$ and $\operatorname{Re}_{S}=19737$. The dashed lines indicate the maxima $\mu_{\max }$ given in Eq. (5). Error bars based on the statistical uncertainty would be smaller than the symbols.

that the torque maximization at $\mu_{\max }(0.71)=-0.357 \pm 0.060$ falls in line with the experimental observations $\mu_{\max }(0.716)=$ $-0.33 \pm 0.04$ and $\mu_{\max }(0.7245)=-0.333[10,12]$. Furthermore, the torque maximum in a wide gap Taylor-Couette system has been experimentally confirmed to remain located at $\mu_{\max }(0.5)=-0.20 \pm 0.02$ up to shear Reynolds numbers of $5 \times 10^{5}$ [14].

A final data point is provided by Wendt's data [15], which are reanalyzed in the way used in the recent experiments in the Appendix. For the radius ratio of $\eta=0.680$, the maximum in his torque data lies near

$$
\mu_{\max }(0.680)=-0.295 \pm 0.113 .
$$

\section{ONSET OF THE RADIAL INHOMOGENEITY}

In order to connect the onset of fluctuations with the rotation ratio for maximal torque and its dependence on the radius ratio, we ask the following questions: (i) What is the physical mechanism that determines the onset of fluctuations, and can one derive a prediction for the onset from it? (ii) Why should the rotation ratio of torque maximization coincide with the onset of fluctuations?

One answer to these questions is provided by van Gils et al. [10]: They suggest that the rotation ratio for maximal torque is determined by locations in parameter space $\left(\operatorname{Re}_{i}, \operatorname{Re}_{o}\right)$ that are equally distant from the Rayleigh stability lines $\mu=\eta^{2}$ and $\mu=-\infty$. This condition results in the so-called "angle bisector,"

$$
\mu_{\text {bis }}(\eta)=\frac{-\eta}{\tan \left[\frac{\pi}{2}-\frac{1}{2} \arctan \left(\eta^{-1}\right)\right]}
$$

for the location of the torque maximum [10]. Moreover, they argue that the onset of fluctuations has to coincide with the torque maximum since the intermittent behavior in the outer layer reduces radial transport of momentum so that the torque starts to drop. Although the angle bisector agrees well with their measured maximum $\mu_{\max }(0.716)=-0.33$, it disagrees with our simulation result for $\eta=0.5$ since $\mu_{\max }(0.5)=$ -0.195 , whereas, Eq. (8) gives $\mu_{\text {bis }}(0.5)=-0.309$. 
Here, we propose an explanation for the onset of fluctuations that is not based on the stability of laminar flow but on properties of turbulent flows, in general, and turbulent Taylor vortices, in particular. The key idea is that the turbulent flow detaches because the inner part that is driven by the Rayleigh-unstable region is not sufficiently strong to maintain persistent turbulence across the Rayleigh-stable region to the outer cylinder. But the outer region cannot return to laminar for all times because the turbulent transport and the friction have to be the same, independent of the radial position [20]. The radial range over which the inner unstable region can maintain a turbulence is somewhat larger than the inner Rayleigh-unstable region. The simple inviscid stability calculations for counterrotation alluded to before give a neutral surface at radius,

$$
r_{n}(\mu)=r_{i} \sqrt{\frac{1-\mu}{\eta^{2}-\mu}},
$$

that separates stable from unstable flow [21] and implies a detachment of the unstable flow for any $\mu<0$. However, experiments and viscous calculations show that Taylor vortices extend beyond this neutral surface when counter-rotation sets in, see, for example, Ref. [1]. Esser and Grossmann [22] deduced, from their stability calculation, that flow structures protrude the neutral surface by a factor of $a(\eta)$, i.e., the effective extension of secondary flow is

$$
r_{\mathrm{EG}}(\mu)=r_{i}+a(\eta)\left(r_{n}-r_{i}\right)
$$

with a factor,

$$
a(\eta)=(1-\eta)\left[\sqrt{\frac{(1+\eta)^{3}}{2(1+3 \eta)}}-\eta\right]^{-1},
$$

that takes values between 1.4 and 1.6. The rotation ratio $\mu_{\text {pred }}$ where the unstable flow detaches from the outer cylinder wall then follows from the condition $r_{\mathrm{EG}}\left(\mu_{\text {pred }}\right)=r_{o}$ and reads

$$
\mu_{\text {pred }}(\eta)=-\eta^{2} \frac{\left(a^{2}-2 a+1\right) \eta+a^{2}-1}{(2 a-1) \eta+1} .
$$

For $\mu<\mu_{\text {pred }}$, the turbulence can no longer fill the whole cylinder gap, i.e., $r_{\mathrm{EG}}<r_{o}$, and intermittency has to set in. The extended range of the inner unstable region as captured by the factor $a(\eta)$ then mandates a minimal counter-rotation for the neutral surface to fall inside the cylinders. The evaluation of (12) yields predictions $\mu_{\text {pred }}(0.5)=-0.191$ and $\mu_{\text {pred }}(0.71)=$ -0.344 that compare well with the empirically found onsets of intermittency (4), see also Table I.

\section{ENHANCED LARGE-SCALE CIRCULATION}

We now turn to the question of why the torque maximum coincides with the onset of the radial intermittency. van Gils et al. [10] argue that the torque decreases when turbulent bursting sets in because of the reduced radial transport. However, to obtain a torque maximum at the bursting onset $\mu_{c}$, additionally, the torque has to increase with $\mu$ decreasing from zero to $\mu_{c}<0$. We argue that this increase is caused by a strengthening of the mean Taylor vortex flow. Such a large-scale circulation (LSC) is able to effectively transport momentum and, thus, to increase the torque in addition to turbulent fluctuations. The strengthening of the LSC is
TABLE I. Rotation ratio of the onset of intermittency $\mu_{c}$ and of the torque maximum $\mu_{\max }$ together with the new prediction $\mu_{\text {pred }}$ (12) and the angle bisector $\mu_{\text {bis }}(8)$ for various radius ratios. The rotation ratio $\mu_{\mathrm{LSC}}$ of the maximal mean-flow contribution to the torque is also given for the numerical simulations in the last column.

\begin{tabular}{lccccc}
\hline \hline$\eta$ & $\mu_{c}$ & $\mu_{\max }$ & $\mu_{\text {pred }}$ & $\mu_{\text {bis }}$ & $\mu_{\text {LSC }}$ \\
\hline 0.5 & -0.208 & -0.195 & -0.191 & -0.309 & -0.223 \\
0.68 & & -0.295 & -0.321 & -0.360 & \\
0.71 & -0.325 & -0.357 & -0.344 & -0.367 & -0.357 \\
0.716 & -0.368 & -0.33 & -0.349 & -0.368 & \\
0.7245 & & -0.333 & -0.356 & -0.370 & \\
\hline \hline
\end{tabular}

due to a change in the effective outer boundary condition: Whereas, for $\mu>\mu_{\text {pred }}$, the LSC seeks to extend beyond the outer cylinder and is restricted by the rigid wall, i.e., $r_{\mathrm{EG}}>r_{o}$, the rigid boundary conditions become replaced by a softer free-surface-like laminar outer boundary layer as $\mu$ approaches $\mu_{\text {pred }} \sim \mu_{c}$. Less restricted, the large-scale vortices can become stronger before they will be destroyed by the bursting for $\mu<\mu_{c}$.

To test this picture, we decompose the flow into the LSC contribution $\overline{\mathbf{u}}=\langle\mathbf{u}\rangle_{\varphi, t}$ that contains the mean variations in radial and axial directions and the turbulent fluctuations $\mathbf{u}^{\prime}=$ $\mathbf{u}-\overline{\mathbf{u}}$. With this decomposition, from Eq. (2), one finds a partitioning of the torque,

$$
G=\bar{G}+G^{\prime}
$$

with the mean-flow (LSC) and turbulent contributions,

$$
\begin{aligned}
\bar{G} & =v^{-2}\left\langle r^{3}\left(\left\langle\bar{u}_{r} \bar{\omega}\right\rangle_{A(r), t}-v \partial_{r}\langle\bar{\omega}\rangle_{A(r), t}\right)\right\rangle_{r}, \\
G^{\prime} & =v^{-2}\left\langle r^{3}\left\langle u_{r}^{\prime} \omega^{\prime}\right\rangle_{A(r), t}\right\rangle_{r} .
\end{aligned}
$$

The mixed terms $\left\langle\bar{u}_{r} \omega^{\prime}\right\rangle$ and $\left\langle u_{r}^{\prime} \bar{\omega}\right\rangle$ in (2) as well as $\left\langle\omega^{\prime}\right\rangle_{A(r), t}$ vanish due to the definition of $\overline{\mathbf{u}}$. Although the complete torque $G$ is radially independent, similar expressions for mean-flow and turbulent contributions vary with the radius. Therefore, we introduced an additional radial average in (14) to measure the mean weight of each contribution. Moreover, to accurately capture the mean Taylor vortex motion, we axially shift the instantaneous flow fields during the temporal average of $\overline{\mathbf{u}}$ and in (14) so that Taylor vortices always stay at a fixed height.

For $\mu \gtrsim 0$, the torque is mainly caused by turbulent fluctuations, and the mean-flow contribution nearly drops to the laminar level as shown in Fig. 4. Turbulent fluctuations also dominate the torque for strong counter-rotation, i.e., $\mu \lesssim-0.5$ for $\eta=0.5$ and $\mu \lesssim-0.71$ for $\eta=0.71$. For intermediate rotation ratios, mean-flow vortices contribute the major share to the torque. Note that the onset of mean vortical flow for $\mu>$ $-\eta$, with $\mu=-\eta$ corresponding to perfect counter-rotation $\operatorname{Re}_{o}=-\mathrm{Re}_{i}$, was previously observed by Ravelet et al. [23]. In addition, the LSC contribution grows with $\mu$ decreasing from zero, which is consistent with our picture of a change in the outer boundary condition from no-slip to a less restrictive free-surface condition. The mean Taylor vortices are strongest (as measured by their contribution to the torque) at the rotation ratio $\mu_{\mathrm{LSC}}(\eta)$ where $\bar{G}$ is maximized. Using a quadratic fit with 
(a)

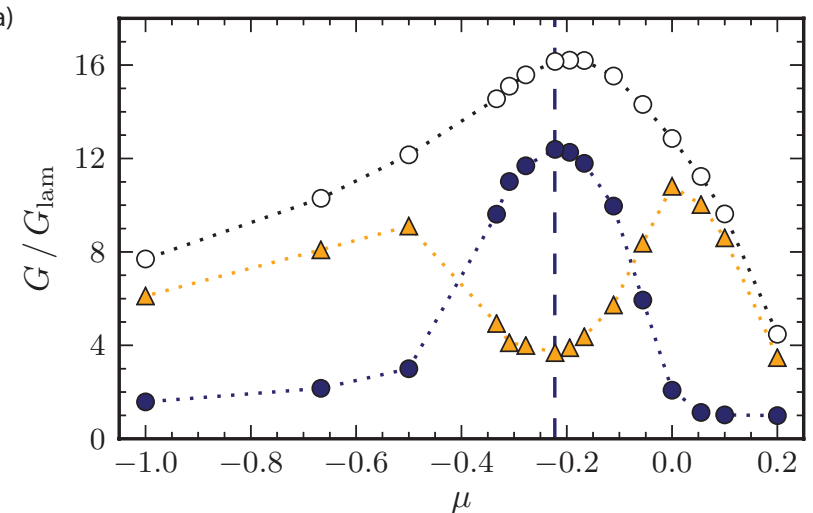

(b)

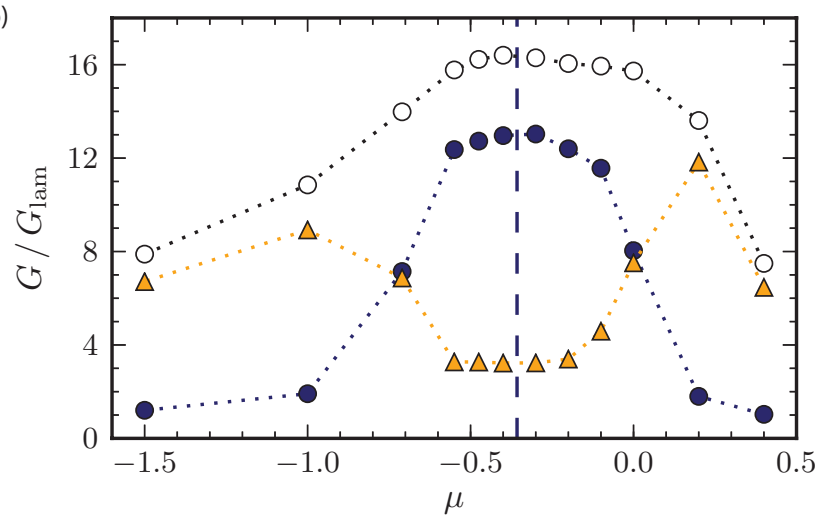

FIG. 4. (Color online) Rotation dependence of the contributions to the torque. Dark circles (blue): mean-flow contribution. Gray triangles (orange): turbulent contribution. Open circles: complete torque. The torque due to the mean flow exhibits a maximum at $\mu_{\text {LSC }}$ marked by the dashed line and given in Eq. (15). Dotted lines connecting the data serve as a guide to the eye. (a) $\eta=0.5$ and $\operatorname{Re}_{S}=20000$. (b) $\eta=0.71$ and $\operatorname{Re}_{S}=19737$.

an uncertainty estimate analogous to (6), we find

$$
\begin{aligned}
\mu_{\mathrm{LSC}}(0.5) & =-0.223 \pm 0.018, \\
\mu_{\mathrm{LSC}}(0.71) & =-0.357 \pm 0.075 .
\end{aligned}
$$

Consequently, the rotation ratio of optimal momentum transport by the mean flow coincides with the empirically found onset of intermittency Eq. (4) within the given uncertainties. Furthermore, the mean-flow contribution is responsible for the maximum in the total torque, cf. Fig. 4 and Eq. (5), thereby, establishing the connection between the onset of intermittency and the torque maximum within the framework depicted above.

This connection implies that the prediction for the intermittency onset also acts as a prediction for torque maxima. We, thus, compare the predictions from the boundary layer argument $\mu_{\text {pred }}(\eta)$ from Eq. (12) and the bisection argument $\mu_{\text {bis }}(\eta)$ from Eq. (8) [10] with experimental and numerical results for torque maxima in Fig. 5 (and Table I). The rotation ratio $\mu=-2.797$ of the turbulent bursts found by Coughlin and Marcus [8] lies below both predictions and, thus, clearly in the intermittent range. Our simulation result for $\eta=0.71$ and Wendt's experimental result for $\eta=0.680$ are consistent with both the angle bisector and the current prediction within

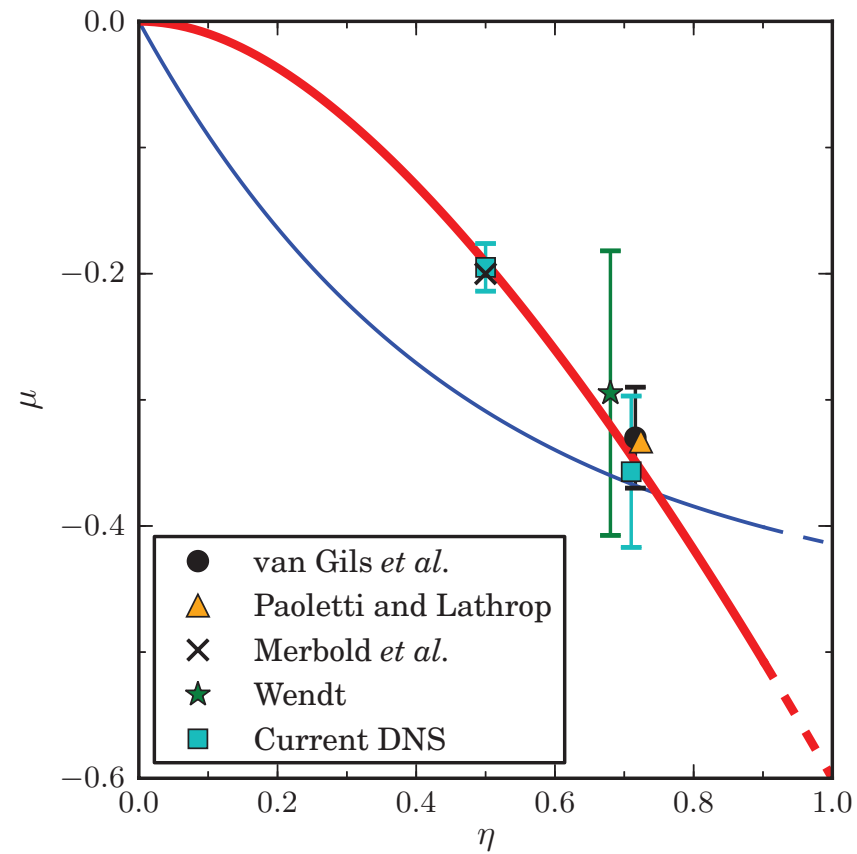

FIG. 5. (Color online) Location of torque maxima for different radius ratios $\eta$. The thin (blue) line is the angle bisector (8), and the thick (red) line is the boundary layer estimate (12). The black circle, orange triangle, black cross, and green star show torque maxima from the experiments [10], [12], [14], and [15]. The cyan squares mark the simulation result given in Eq. (5). Since the predictions are based on the centrifugal instability and the separation into flow regions, which both disappear for $\eta \rightarrow 1$, we indicate the resulting uncertainty for $\eta \gtrsim 0.9$ by a dashed line.

the error bars. Moreover, we note that the torque maximum $\mu_{\max }(0.7245)=-0.333$ measured by Paoletti and Lathrop [12] as well as $\mu_{\max }(0.716)=-0.33 \pm 0.04$ measured by van Gils et al. [10] tend towards our prediction Eq. (12). However, considering the usual error bars, these values are also consistent with the angle bisector line. The empirical results for $\eta=0.5$ provide a better test of both predictions since the values obtained from (8) and (12) differ. The numerical (5) and experimental [14] data are in better agreement with the boundary layer estimate (12).

\section{FINAL REMARKS}

The analysis presented here supports the idea that the torque increases with increasing counter-rotation because the vortices gain in strength until they can no longer sustain turbulence all across the gap. The torque drops for stronger counter-rotation when the detachment of mean vortices from the outer layer leads to radial intermittency.

The boundary layer argument presented here and the angle bisection proposal of van Gils et al. [10] give indistinguishable predictions for a radius ratio of $\eta \approx 0.75$, but the shape of the $\eta$ dependencies is sufficiently different that data, in particular for smaller $\eta$, should allow for distinguishing between the two. The available data for $\eta=0.5$ are in good agreement with the present argument. Clearly, results for more $\eta$ are required, and work along those lines is in progress. 


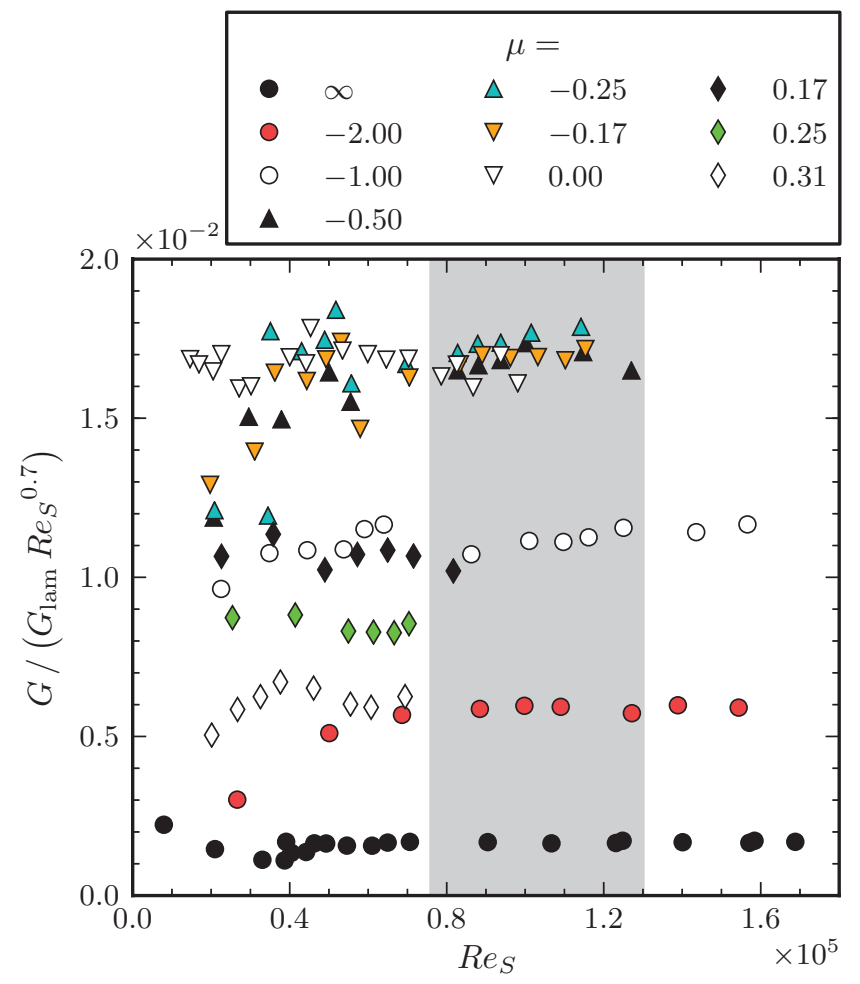

FIG. 6. (Color online) Torques measured by Wendt [15] for $\eta=$ 0.680 and various rotation ratios. The values are compensated by the laminar $G_{\text {lam }}$ and by the effective scaling $G / G_{\text {lam }} \sim \operatorname{Re}_{S}^{0.7}$ reported by Wendt. The torques for the range shaded in gray are further analyzed in Fig. 7.

For larger $\eta$ in the limit $\eta \rightarrow 1$, the current theory predicts a maximum for a rotation ratio of -0.6 , whereas, the angle bisection gives a value close to -0.4 . However, this limit is delicate because the linearly unstable region disappears and the transition to turbulence follows a different route [24]. In this case, both theories will most likely have to be refined or will have to be replaced. Evidence for this is provided, for instance, by the measurements by Ravelet et al. [23] for $\eta=0.917$, which do not show a torque maximum for counter-rotation. Figure 5 reflects this uncertainty in the prediction by the change from a continuous to a dashed line for $\eta>0.9$.

Furthermore, numerical simulations revealed that, at lower $\operatorname{Re}_{S} \leqslant 4 \times 10^{3}$, the torque is maximized at $\mu \approx 0$ for $\eta=$ 0.71 [13]. This larger rotation ratio is not covered by the current theory so that further refinements are needed for lower Reynolds numbers and mildly turbulent flows.

\section{ACKNOWLEDGMENTS}

We are grateful to M. Avila for developing and providing the code used for our simulations. This work was supported, in part, by the Deutsche Forschungsgemeinschaft within the research group FOR1182. Most computations were performed at the LOEWE-CSC in Frankfurt.

\section{APPENDIX: REANALYSIS OF WENDT'S DATA}

Recent experimental papers analyze the dependence of torque on the shear rate and on the mean system rotation

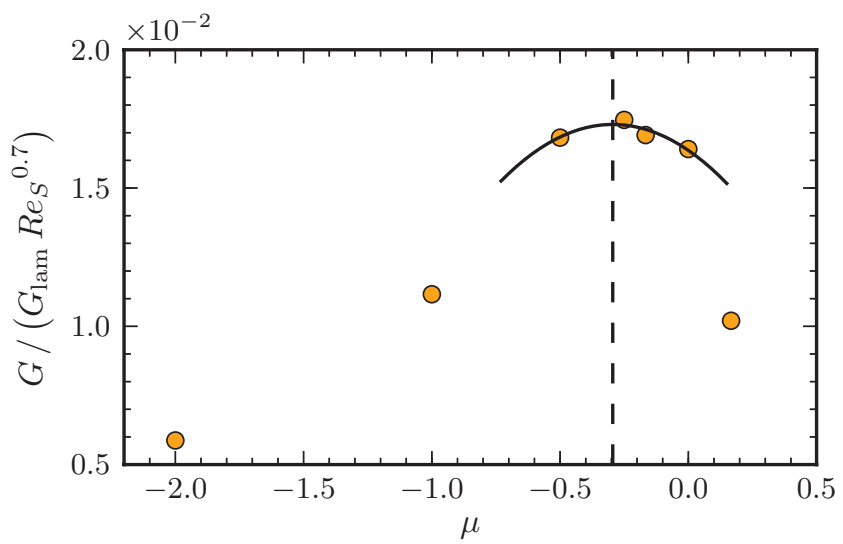

FIG. 7. (Color online) Compensated torques by Wendt for $\eta=$ 0.680 independently averaged for each rotation ratio in the range of $7.6 \times 10^{4}<\operatorname{Re}_{S}<1.3 \times 10^{5}$. The solid line indicates a quadratic least-squares fit to the four largest values. Its maximum $\mu_{\max }=$ -0.295 is marked by the dashed line.

independently. This decomposition is advantageous since torques can be compensated either by dividing by the effective scaling with the shear [11] or by taking the ratio to $G(\mu=0)$ $[12,17]$ to study the rotation dependence. The resulting torque amplitudes are based on numerous measurements at different shear rates for each rotation ratio, which improves statistical significance. In contrast, Wendt presented the dependence of the torque on the rotation for some selected shear Reynolds numbers in Fig. 10 of Ref. [15]. Since this evaluation is based on single measurements, uncertainties may play a major role.

Therefore, here, we apply the current analysis method to Wendt's torque measurements for $\eta=0.680$ digitized from Fig. 9 in Ref. [15]. Figure 6 shows the torques for various rotation ratios compensated by $\operatorname{Re}_{S}^{0.7}$, which Wendt found as effective scaling for $10^{4} \lesssim \operatorname{Re}_{S} \lesssim 10^{5}$. One easily sees that the torque depends on the mean rotation with the largest values for high $\operatorname{Re}_{S}$ at $\mu=-0.25$. We closely follow the analysis in Refs. [10-12] and average the compensated torques in the range of $7.6 \times 10^{4}<\operatorname{Re}_{S}<1.3 \times 10^{5}$ to find amplitudes depending on the mean system rotation only, see Fig. 7. We choose this Reynolds number range so that it starts after the shift in the torque maximum [13] and includes the highest data points for $-0.50 \leqslant \mu \leqslant 0.17$ (cf. Fig. 6). One observes a maximum in the statistically more significant mean amplitudes for moderate counter-rotation, which was also found in recent papers [1012] and in current simulations. Based on a quadratic fit to the largest amplitudes, we find

$$
\mu_{\max }(0.680)=-0.295 \pm 0.113
$$

with the uncertainty calculated in analogy to Eq. (6). Its relatively high level is due to the broad maximum in Fig. 7 and due to the few rotation ratios investigated by Wendt. Despite the high uncertainty, the torque maximization for counter-rotation, i.e., $\mu_{\max }<0$, is clear without ambiguity. Moreover, the new maximum $\mu_{\max }(0.680)=$ -0.295 lies consistently between the maxima identified here, cf. Eq. (5). 
[1] G. I. Taylor, Philos. Trans. R. Soc. London, Ser. A 223, 289 (1923).

[2] E. L. Koschmieder, Bénard Cells and Taylor Vortices (Cambridge University Press, Cambridge, U.K., 1993).

[3] C. D. Andereck, S. S. Liu, and H. L. Swinney, J. Fluid Mech. 164, 155 (1986).

[4] D. Coles, J. Fluid Mech. 21, 385 (1965).

[5] C. Van Atta, J. Fluid Mech. 25, 495 (1966).

[6] A. Meseguer, F. Mellibovsky, M. Avila, and F. Marques, Phys. Rev. E 80, 046315 (2009).

[7] S. Dong and X. Zheng, J. Fluid Mech. 668, 150 (2011).

[8] K. Coughlin and P. S. Marcus, Phys. Rev. Lett. 77, 2214 (1996).

[9] P. W. Colovas and C. D. Andereck, Phys. Rev. E 55, 2736 (1997).

[10] D. P. M. van Gils, S. G. Huisman, S. Grossmann, C. Sun, and D. Lohse, J. Fluid Mech. 706, 118 (2012).

[11] D. P. M. van Gils, S. G. Huisman, G.-W. Bruggert, C. Sun, and D. Lohse, Phys. Rev. Lett. 106, 024502 (2011).

[12] M. S. Paoletti and D. P. Lathrop, Phys. Rev. Lett. 106, 024501 (2011).
[13] H. J. Brauckmann and B. Eckhardt, J. Fluid Mech. 718, 398 (2013).

[14] S. Merbold, H. J. Brauckmann, and C. Egbers, Phys. Rev. E 87, 023014 (2013).

[15] F. Wendt, Ing. Arch. 4, 577 (1933).

[16] A. Meseguer, M. Avila, F. Mellibovsky, and F. Marques, Eur. Phys. J.: Spec. Top. 146, 249 (2007).

[17] B. Dubrulle, O. Dauchot, F. Daviaud, P.-Y. Longaretti, D. Richard, and J.-P. Zahn, Phys. Fluids 17, 095103 (2005).

[18] D. P. Lathrop, J. Fineberg, and H. L. Swinney, Phys. Rev. A 46, 6390 (1992).

[19] D. P. Lathrop, J. Fineberg, and H. L. Swinney, Phys. Rev. Lett. 68, 1515 (1992).

[20] B. Eckhardt, S. Grossmann, and D. Lohse, J. Fluid Mech. 581, 221 (2007).

[21] S. Chandrasekhar, Hydrodynamic and Hydromagnetic Stability, 1st ed. (Clarendon, Oxford, 1961).

[22] A. Esser and S. Grossmann, Phys. Fluids 8, 1814 (1996).

[23] F. Ravelet, R. Delfos, and J. Westerweel, Phys. Fluids 22, 055103 (2010).

[24] H. Faisst and B. Eckhardt, Phys. Rev. E 61, 7227 (2000). 